\title{
Study on an Information Processing Model with Psychological Memory for a Kansei Robot
}

\author{
Yuta Kita ${ }^{1}$, Masataka Tokumaru ${ }^{2}$, and Noriaki Muranaka² \\ ${ }^{1}$ Graduate School of Kansai University, 3-3-35 Yamate-cho, Suita-shi, \\ Osaka 564-8680, Japan \\ k366457@kansai-u.ac.jp \\ ${ }^{2}$ Kansai University, 3-3-35 Yamate-cho, Suita-shi, Suita, Osaka 564-8680, Japan
}

\begin{abstract}
In this paper, we propose an information processing model for a kansei robot. This model handles memory based on human psychology. We expect that on incorporating the model, a robot can exhibit human characteristics because of using psychological memory. To verify the model, we first perform a comparison between the results of the experiment performed using this model and that of an actual psychological experiment. The results of the comparison suggest that the memory functions of the model are similar to the human memory functions. Second, we conduct the process of learning movement actions to verify that the robot on which the model was implemented learned movement for moving to many places and decreasing its curiosity.
\end{abstract}

Keywords: kansei, robot, memory, neural network, psychology.

\section{Introduction}

For making partner robots resemble humans they need to have human characteristics. However, the method to instill such characteristics in robots is not known yet. Therefore, we suggest an information processing model based on human psychology [1]. This model is constructed by referring to information processing models [2]. The model explains how internal or external information is handled. In this study, we verify the usability of the model, particularly for memory.

\section{Information Processing Model}

Figure.1 shows the information processing model we propose. First, data captured by the sensors of a robot enters the Sensory Memory and then, the data is transferred to the Short Memory only if the robot observes (Observe, a robot action). Second, the robot's action is generated using a multilayer neural network (NN), whose inputs are the input data in the Short Memory and the robot's internal conditions (e.g., desire).

In addition, the indexes of forgetting and remembering memory data are given to the Short Memory and the Episodic Memory. We apply the function proposed by Wayne A. Wickelgren [3] to the index of the Episodic Memory. 


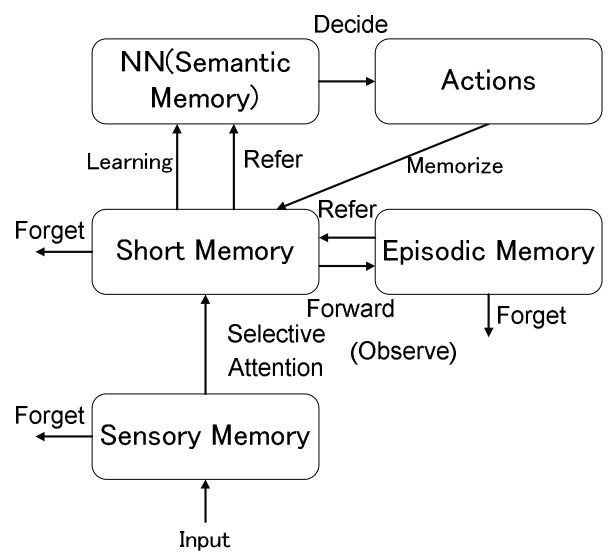

Fig. 1. Information processing model

Here, we define the want values which are the values of the robot's internal conditions. The want values steadily increase; they decrease only if a robot's action succeeds. When the robot learns something, the NN is given the spread coefficient referring to the rate of the decrease in the want value. However, this learning is executed only when a robot's action ends, and there is a possibility that this learning is inadequate. Therefore, a robot executes the offline learning when the input data in the Short Memory is similar to data in the Episodic Memory (Recall under Cued).

We performed a simulation for verifying that the memory function of the model is similar to the human memory function. This simulation is a comparison between the outcome of the model and that of the experiment conducted by Bennet B. Murdock [4]. The results indicate that their similarity is $90.6 \%$; thus we assumed that their functions are similar.

\section{Learning the Movement Actions Simulation}

From the simulation, we can determine the processes for learning the movement actions. Here, there are six robot actions, generated by the recurrent NN; turn left and right, move forward and backward, observe, and do nothing. The robot's want value is referred to as curiosity. Equation (1) shows how to decrease its curiosity if a robot moves.

$$
\text { Curiosity }_{k}=\text { Curiosity }_{k-1} \times(0.5+0.5 \times \operatorname{Sim}(\text { position })) \text {. }
$$

Here, Sim(position) is the function, which returns the similarity between position information of memory and that of the robot's position; Lesser the memory a robot has regarding the place, the more its curiosity decrease.

Figure. 2 shows the path (indicated by arrows) of a round robot moving in a square room with action steps. The figure also shows that the darker the shade of the path is, the more frequent the robot is present at that location. We assumed that the robot 


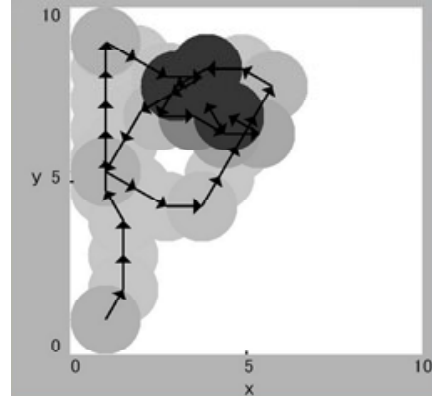

(a) 0-500 steps

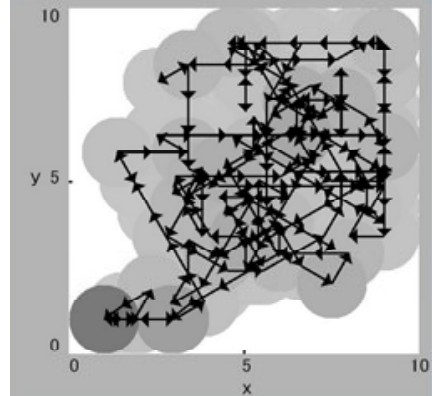

(b) 1000-1500 steps

Fig. 2. State of the moving round robot

learned the movement actions needed to travel throughout the room to decrease its curiosity, because the robot had moved a considerable number of steps.

\section{Conclusions}

In this paper, we proposed an information processing model for a kansei robot. In addition, we performed a comparison of the results of this study with the experiment that Bennet B. Murdock conducted, which indicated that the memory function in the memory and the human memory function have a similarity is $90.6 \%$. The second simulation for learning the movement actions indicated that the robot moved to decrease its curiosity.

This study showed that the robot exhibited human characteristics. However, we still need to verify the features of an actual robot because an experiment with an actual robot was not conducted.

\section{References}

1. Inoue, K., Tokumaru, M., Muranaka, N.: Considering the Characteristics of Living Creatures for Kansei Robot Action Generation Using a Neural Network. In: Joint 4th International Conference on Soft Computing and Intelligent Systems and 9th International Symposium on Advanced Intelligent Systems, pp. 50-55 (2008)

2. Baddeley, A.: Working Memory, Thought, and Action. Oxford University Press, USA (2007)

3. Wickelgren, W.: Single-trace fragility theory of memory dynamics. Memory \& Cognition 2(4), 775-780 (1974)

4. Murdock Jr., B.: The serial position effect of free recall. Journal of Experimental Psychology 64, 482-488 (1962) 